\title{
Modulation of grip force with load force during point-to-point arm movements
}

\author{
J. Randall Flanagan, Alan M. Wing \\ MRC Applied Psychology Unit, 15 Chaucer Road, Cambridge CB2 2EF, UK
}

Received: 4 December 1992/Accepted: 12 March 1993

\begin{abstract}
In this paper, we examine grip forces and load forces during point-to-point arm movements with objects grasped with a precision grip. We demonstrate that grip force is finely modulated with load force. Variations in load force arise from inertial forces related to movement; grip force rises as the load force increases and falls as load force decreases. The same finding is observed in vertical and horizontal movements performed at various rates. In vertical movements, maximum grip force coincides in time with maximum load force. The maxima occur early in upward and later in downward movements. In horizontal movements, where peaks in load force are observed during both the acceleratory and deceleratory phases, grip force rises at the beginning of the movement and remains high until the end. The results suggest that when moving an object with the hand the programming of grip force is an integral part of the planning process.
\end{abstract}

Key words: Motor Control - Grip force - Precision grip - Arm movement - Human

\section{Introduction}

There have been a number of demonstrations of grip force changes that occur in anticipation of environmental demands. For example, when an object is lifted, grip force increases simultaneously with load force prior to lift-off (Johansson and Westling 1984). The rate of increase of grip force and the final grip force depend on the object's weight and its surface texture. Anticipatory increases in grip force can also be observed when subjects see a ball being dropped into a cup that they are supporting (Johansson and Westling 1988). The change in grip force anticipates the increase in load force that will result from contact between ball and cup and guards against slippage. Anticipatory grip force adjustments may be contrasted with reflex-mediated changes in grip force that

Correspondence to: J. R. Flanagan arise, after a delay, when unexpected loads are applied to grasped objects (Johansson and Westling 1987; Cole and Abbs 1988).

The coordination of grip force and load force during lifting and holding has been described in detail by Johansson and Westling $(1984,1988)$. These researchers have focused on three phases of the lift: preload, loading and hold. During the initial preload phase, grip force begins to increase (without a change in load force) as the grasp is established. In the subsequent loading phase, grip force and load force increase simultaneously until the load force exceeds the weight of the object and lift-off occurs. This is followed by the holding phase where the object is held aloft.

Johansson and Westling (1984) have reported that, during the loading phase, the maximum rate of change of grip force depends on the final grip force at lift-off (and during the subsequent holding phase). The greater the final grip force, the larger the rate of change. Because the final grip force was found to be higher for heavier and more slippery objects, Johansson and Westling concluded that increases in grip force during the loading phase are planned in anticipation of the properties of the object.

The excess grip force (i.e. the observed grip force minus the minimum grip force required to prevent slippage) is also greater for heavy and slippery objects. Indeed, Johansson and Westling (1984) have observed that the ratio of excess to minimum grip force is approximately constant across all objects. They have suggested that the excess grip force guards against random fluctuations in the motor system and that grip force is scaled with load force in order to economize effort. In other words, the rule seems to be "do not use more force than necessary above some safety margin."

Previous work has shown that anticipatory changes in grip force are sensitive to environmental demands related to the characteristics of the grasped object. In this paper we ask how grip force is modulated when the properties of the object are kept constant but subjects are asked to move the object about. When a grasped object is moved, a force (proportional to acceleration) must be applied to 
overcome its inertia. At the same time, if the object is grasped with a precision grip (i.e. with the tips of the thumb and index finger on its sides), the grip force must be high enough to prevent slippage. Because the friction between the digits and the object depends on grip force, increases in grip force enable the hand to apply larger load forces without slippage. The questions arise as to how grip force is modulated during arm movements to cope with changes in load force induced by the movement and which mechanisms are responsible.

Changes in grip force have been shown to anticipate changes in load force induced by movements against spring and stiff loads. Johansson and Westling (1984) demonstrated that grip force and load force change in parallel when subjects move a spring-loaded object where the load force increases with displacement. More recently, Johansson et al. (1992) reported that changes in grip force parallel changes in load force when pulling on a stiff, fixed object where the load depends on the isometric force exerted by the hand. However, the coupling between grip force and load force during arm movements with inertial loads has not been systematically documented.

It would seem unlikely that the motor system could rely on reflex mechanisms to control grip forces during voluntary arm movements, especially in fast movements where there are large and rapid fluctuations in load force due to inertial loading. When a brief and unexpected load force is applied to a grasped object, an increase in grip force is observed 60-90 ms later (Johansson and Westling 1987; Cole and Abbs 1988). This is about the time expected for a supraspinal sensorimotor reflex loop. The average velocity of the hand in a fast arm movement can be well above $1 \mathrm{~m} / \mathrm{s}$. At this rate, if inertial force caused the object to slip, the hand would move at least $6 \mathrm{~cm}$ by the time a reflexive increase in grip force (invoked by slippage) would appear. Thus, it is likely that the object would be dropped. Given the delays associated with reflex mechanisms, one might reasonably expect to see anticipatory grip force adjustments, during movements, to deal with the inertial forces that arise.

There is evidence, from a number of other tasks, of preparatory actions that accommodate forces that arise from kinematics. For example, anticipatory adjustments to cope with intersegmental forces resulting from arm movement have been reported in remote body segments as well as within the same limb. Horak et al. (1984) have shown that when subjects raise their arm, activity in trunk and leg muscles precedes the arm movement. When the subject was asked to move faster or to lift heavier loads, these postural muscles became active earlier relative to the onset of arm displacement. Bouisset and Zattara (1987) have argued that postural adjustments create forces which compensate for the forces that will be produced by the upcoming arm movement. Smeets et al. (1990) have reported that in rapid elbow flexion movements against loads, shoulder and elbow muscles are activated at about the same time. Even though the shoulder does not move appreciably, clear agonist muscle activity is seen in the shoulder flexors. This produces a flexor torque at the shoulder that compensates for interaction torques, resulting from the elbow movement, that act in extension.

Several ways in which grip force might be modified during movement may be entertained. One possibility is that the grip force increases at the start of the movement, up to a steady level high enough to prevent slippage, and then decreases at the end of the movement. The appropriate grip force level could be derived from the maximum load force predicted for the arm movement. A second possibility is that grip force is tightly coupled with load force such that grip force is modulated in parallel with load force during the movement. In this case, we would expect changes in grip force to depend on the pattern of load force modulation and, hence, the direction of movement. For example, in horizontal movements (where inertial and gravitational forces act orthogonally), peaks in load force are observed during the acceleratory and deceleratory phases. Thus, two grip force peaks would be expected. In contrast, vertical movements (where inertial and gravitational loads act in the same direction) generally exhibit a single load force peak. This occurs near the start of upward movements and towards the end of downward movements. Thus, a single grip force peak would be expected near the start and end of upward and downward movements, respectively. Of course, alternatives lying between these extreme possibilities can be considered. For example, grip force might be adjusted for increases, but not decreases, in load force. In this case, grip force would increase when load force increases, but thereafter decline slowly.

In this paper, we investigate the relation between grip force and load force during vertical and horizontal arm movements of varying rate and direction. The plane of motion was varied in order to produce different patterns of load force variation. By varying movement rate, we manipulated the amplitude of inertial load modulation. A brief preliminary report of some of this work has been published (Flanagan et al. 1993).

\section{Materials and methods}

\section{Subjects}

Eleven subjects between 17 and 40 years of age participated in this study. Four different experiments were carried out. Seven of the subjects only participated in the "first trial" experiment (see below). All subjects gave informed consent before participating.

\section{Apparatus}

Subjects grasped a cylindrical force transducer (Novatech, model 241) between the distal pads of the thumb and index finger (see Fig. 1). The mass of the transducer was $0.26 \mathrm{~kg}$ and the width between grip surfaces was $58 \mathrm{~mm}$. The grip surfaces were steel and were attached to the transducer with a flat-head screw that was flush with the surface. The pads of the digits were placed on the screw. The object's centre of mass was located at its geometric centre midway between the contact points of the thumb and index finger. Therefore, forces did not act to rotate the object during steady grasp or during arm movements. The minimum grip forces with which the subjects were able to hold the object without slippage ranged from 

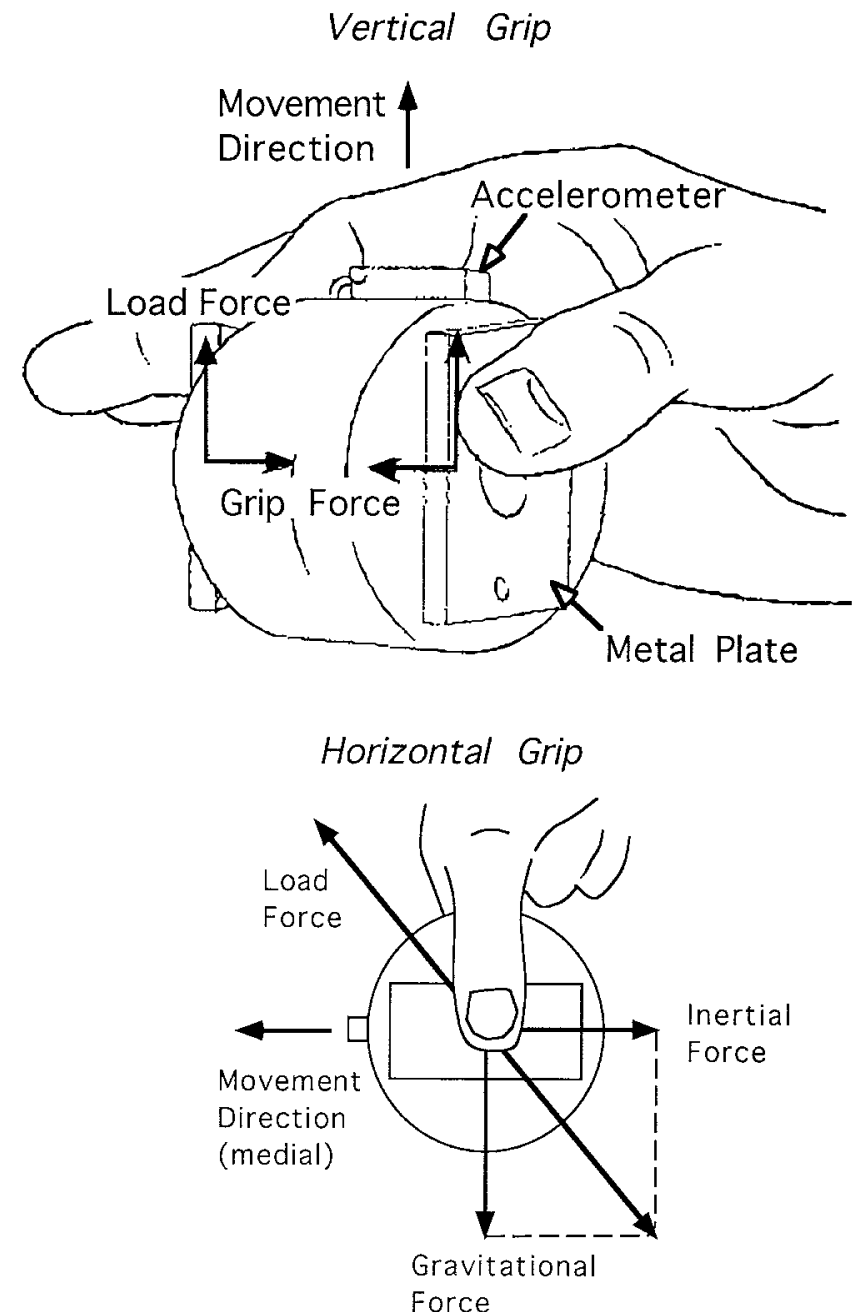

Fig. 1. The force transducer and grasp used in vertical (top) and horizontal (bottom) movements. The same grasp was used in horizontal movements, but the hand and transducer were rotated $90^{\circ}$ about the grip axis with the palm facing down. The direction of the movement relative to the orientation of the grasp was kept constant

2 to 3 N. An accelerometer (Entran, model EGB-125-10D) was mounted on the force transducer and positioned to record acceleration in the direction of movement.

A 16-bit, analog-to-digital interface board (National Instruments, model M10 16X) was used with a Macintosh IIfx computer to collect and store the data. Data collection and analysis were carried out with the LabView graphical programming language (National Instruments).

\section{Experimental procedure}

Subjects held the transducer in front of them while seated. Figure 1 illustrates the grasps adopted for vertical (top) and horizontal (bottom) movements. The orientation of the hand and the transducer relative to the direction of movement was the same in vertical and horizontal movements. In both cases, the grip surfaces were oriented vertically and the load force acted in the plane defined by these surfaces. The direction of movement was approximately normal to the plane defined by the index finger and thumb. Thus, the inertial forces in the vertical and horizontal movements acted in the same direction relative to the hand. However, the direction of the gravitational force with respect to the orientation of the hand was different in vertical and horizontal movements.
The subjects were instructed to move the object in a straight line and to keep the orientation of the object constant during the movement. Subjects were visually monitored by the experimenter throughout the experiment to ensure that they complied with these instructions. (To check that the orientation of the object was kept constant, we used acceleration measurements taken before and after the movement to compute the mean change in angle of the object for each subject-condition combination. The average of the means across conditions and subjects was $5^{\circ}$.) Both the vertical and the horizontal movements were achieved largely by rotations of the upper arm about the shoulder. Small rotations about the elbow and wrist were required to preserve the orientation of the object. Because of the position of the hand in relation to the direction of movement, adjustments at the wrist may have involved medial and lateral deviations, but not flexion and extension. Thus, fluctuations in grip force during the movement were not due to to changes in the length of the long flexors of the hand (see Johansson and Westling 1984).

Experiment 1. In this experiment, the relation between grip and load force during vertical arm movements was examined. Two subjects were instructed to make upward and downward movements at either a moderate rate or a faster rate. The subjects were encouraged to make fairly large amplitude movements. However, targets were not provided. The measured amplitudes varied from 20 to $40 \mathrm{~cm}$. Twenty trials were recorded for each direction and at both movement rates, making 80 trials in all. All trials for a given direction and rate were recorded consecutively. The order in which movements of varying rate and direction were performed was varied.

Experiment 2. The aim of the second experiment was to investigate the coordination of grip force and load force when subjects grasped and moved the transducer for the first time. Seven subjects were asked to pick up the transducer using a precision grip and then move it up and down twice. They were told to move at a comfortable rate and to stop moving between each successive point-topoint movement. None of the subjects had previously performed the task.

Experiment 3. In the third experiment, four subjects were required to produce upward arm movements with the grasped object "aimed" at a maximum load force target (the maximum load force occurs during the initial acceleratory phase of an upward movement). The goal of this experiment was to reduce the load force variability and examine the variability of the grip force. After each trial, load force was displayed as a time series on a monitor along with the target maximum load force. The target load force was 7-9 $\mathrm{N}$, depending on the subject. Subjects had to make fairly fast movements in order to achieve the target maximum load force.

Experiment 4. The final experiment looked at the relation between grip force and load force during medial (to the left for subjects using their right hand) and lateral movements with the transducer held using a precision grip. The same two subjects who participated in experiment 1 were asked to make six to eight medial and lateral movements at a moderate rate and at a faster rate, making a total of 24-32 trials. These movements were similar in amplitude to the vertical movements (i.e. $20-40 \mathrm{~cm}$ ).

\section{Data processing and analysis}

Grip force and acceleration were sampled at $200 \mathrm{~Hz}$. The raw force and acceleration data were then digitally filtered using a fourth-order, zero phase lag, low-pass Butterworth filter with a cutoff frequency of $14 \mathrm{~Hz}$.

The force in the direction of movement was obtained by multiplying the measured acceleration of the object by its mass. In the case of horizontal movements, this force is purely inertial. However, in the case of vertical movements, the force in the direction of 
movement or vertical force is the sum of the inertial force and the force due to gravity (i.e. the weight of the object). The load force was defined as the magnitude of the total or resultant force (inertial and gravitational) acting on the object. Note that in up and down movements the load force is simply the absolute value of the vertical force.

Times to grip force and load force peaks were calculated relative to the start of the movement. The start was taken as the point at which the acceleration exceeded its initial resting level by \pm 2 SDs. (Similarly, the point at which the acceleration reached \pm 2 SDs of the final resting level was taken as the end of the movement.)

In the several plots which show a number of individual force curves, the curves have been aligned at peaks in the load force. It should be noted that the trials shown in some plots are a subset of the total number of trials recorded within a given condition. The trials that are shown were chosen to reflect the typical range of responses.

\section{Results}

We will first describe the results obtained for vertical movements and then focus on those obtained for horizontal movements. Vertical and horizontal movements will then be compared. We will show that in these movements grip force is modulated in phase with load force during the arm movement. In the first two sections, individual and averaged force records are presented and correlations between grip force and load force times and magnitudes are reported. In the final section, different point descriptors are used to compare patterns of grip force modulation in vertical and horizontal movements.

\section{Vertically directed movements}

Qualitative description. Figure 2 shows the grip force and the vertical force acting on the object during a single upward (top panel) and a single downward (bottom panel) movement made at a moderate rate by subject A. Note that the vertical force before and after the movement is equal to the weight of the object $(2.6 \mathrm{~N})$. However, during the movement inertial forces were induced and, consequently, the vertical force fluctuated. In the upward movement, the vertical force exhibited a peak followed by a trough as the object was accelerated up and then down. The opposite pattern was seen in the downward movement. In both cases, the minimum vertical force was close to zero, as the inertial force cancelled out the force due to gravity.

Consider first the upward movement. The example shown in Fig. 2 illustrates that grip force and vertical force increased together over the initial part of the movement and reached their respective maxima at about the same time. The grip force then decreased with vertical force. The increase in grip force with vertical force prevented slippage during the movement. Note that, in this example, the grip force remained elevated during the deceleratory phase of the movement, even though the vertical force dropped to around zero. However, as will be shown below for other subjects, the grip force often dropped below the resting level.

Now consider the downward movement. In contrast

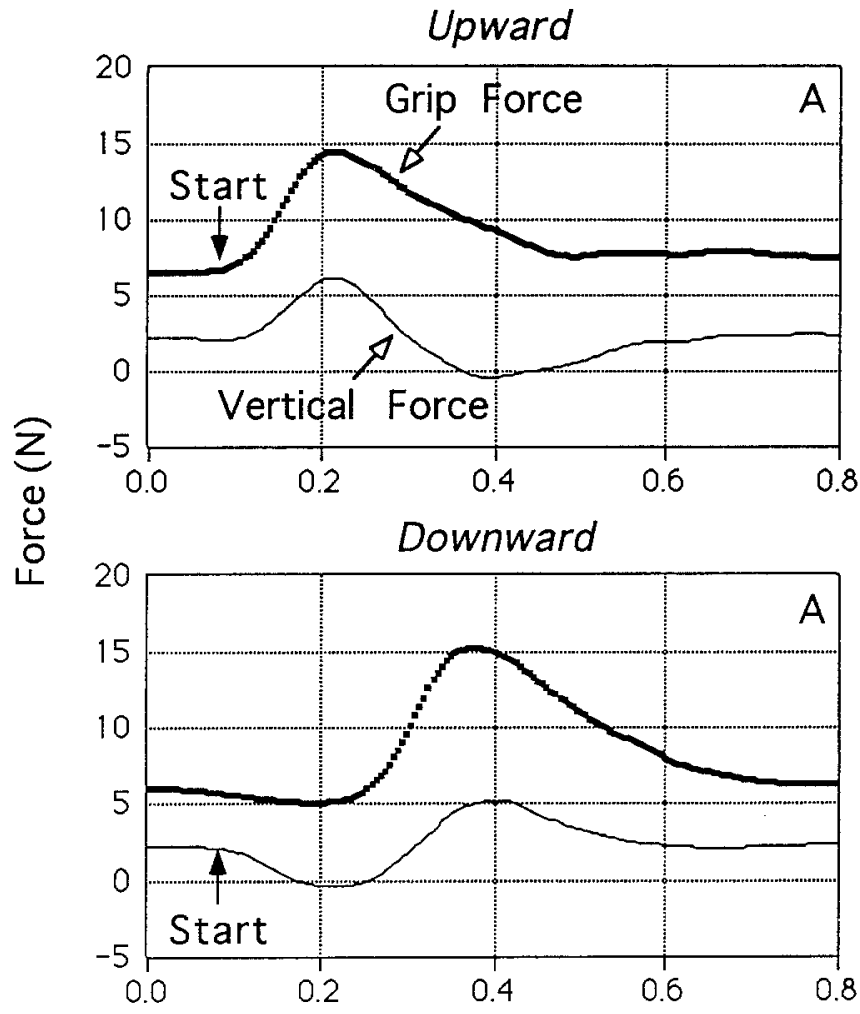

Fig. 2. Grip force and vertical force records of an upward (top panel) and a downward (bottom panel) movement (subject $A$ ). The trials are aligned on movement onset. Grip force increases with vertical force at the start of the upward movement. Parallel increases in grip and vertical force occur later on in the downward movement. Note that the grip force and vertical force maxima coincide closely in time

to the upward movement, the grip force did not increase during the first part of the movement. Indeed, as illustrated in this example, the grip force often decreased as the vertical force fell. An increase in grip force was not necessary here, because the amplitude of the vertical force (i.e. the load force) decreased and there was little danger of the object slipping. The grip force started to rise as the vertical force increased later in the movement. As was the case in the upward movement, the times of the grip force and vertical force maxima coincided and, thereafter, both forces declined together. These two examples illustrate that grip force was modulated in step with vertical force during arm movement. However, the extent to which grip force co-varied with vertical force appears to be less when the latter approached zero. This suggests that the motor system might tend to avoid very low grip forces.

Figure 3 shows grip force and vertical force records of slower upward (left) and downward (right) movements for two subjects (A and B). The trials have been aligned on maximum vertical force. In these two subjects, grip force and vertical force increased and decreased together during both upward and downward movements. Grip force increased at the start of upward and decreased at the start of downward movements, as did the vertical force. In addition, dips in grip force can be seen towards the end of B's movements. In general, the grip force and load force maxima and minima closely coincided in time. 


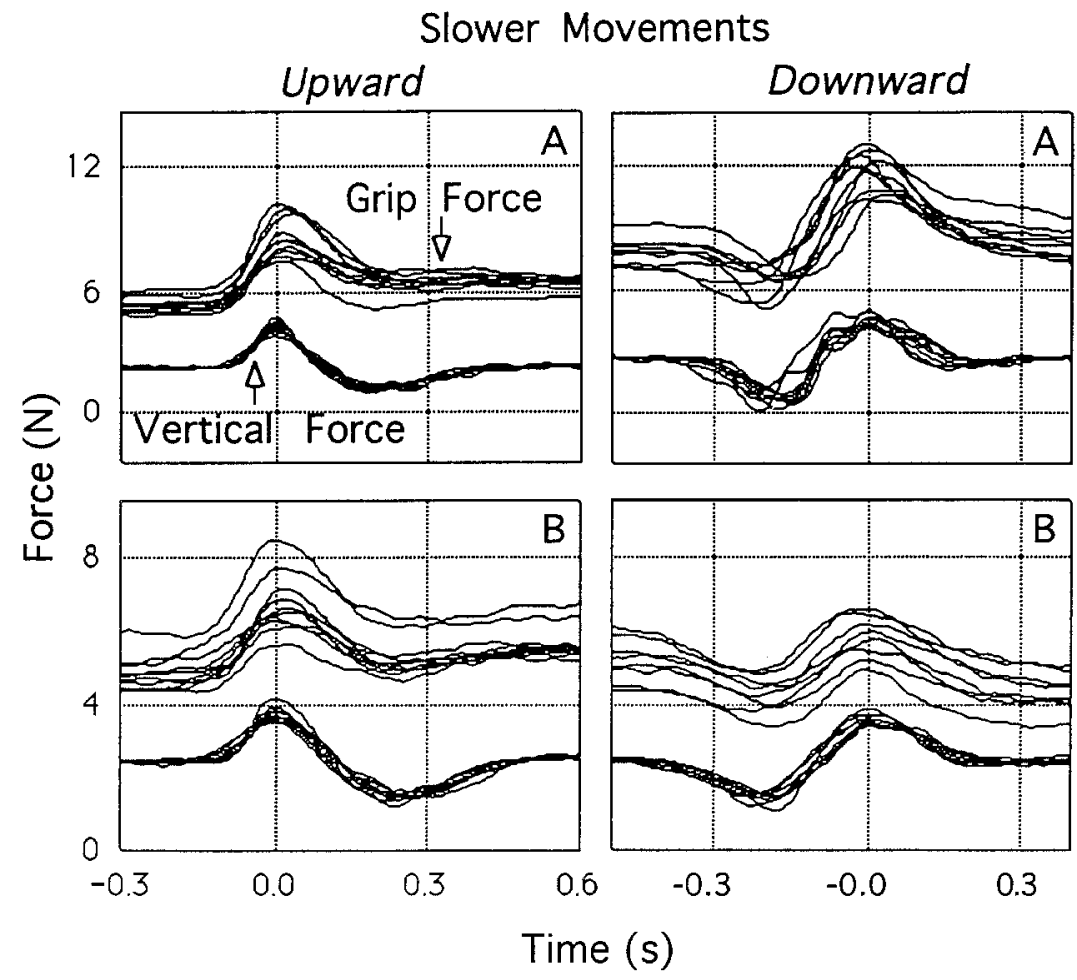

Fig. 3. Grip and vertical force records of slower vertical movements made by two subjects $(A$ and $B$ ). Note that the grip force and vertical force maxima coincide in time, as do the minima. In both subjects, grip force is modulated in phase with vertical force
For subjects A and B the amplitude of grip force modulation appears to be independent of the baseline grip force. Thus, for example, trials that began with a larger grip force featured a larger grip force maximum and ended with a larger grip force. To further examine this issue, the initial and final grip forces were correlated (Pearson product moment) with the maximum grip force. In addition, initial grip force was correlated with the maximum change in grip force relative to the initial level, and final grip force was correlated with the maximum change in grip force relative to the final level. Data from the upward and downward trials were combined. For $A$ and $B$, the initial and final grip forces were significantly correlated with the maximum grip force $(P<0.05)$ but not the maximum change in grip force $(P>0.05)$. These results support the idea that grip force modulation does not vary with baseline grip force. This finding might appear to contradict the results of Johansson and colleague (e.g. Johansson and Westling 1984; Johansson et al. 1992), showing that the ratio of grip force to load force is nearly constant when manipulating objects. However, it should be noted that the variation in baseline grip force we observed was not related to the properties of the object. Had we manipulated the baseline force by varying the surface texture, we would expect to see corresponding changes in maximum grip force.

In Fig. 4, grip force and vertical force functions are shown for faster upward and downward movements made by the same two subjects (A and B). Note that the force and time ranges in these plots are different from those used for slower movements in Fig. 3. The figure shows that, as in slower movements, the grip force and vertical force maxima occurred at about the same time. In the upward movements, grip force increased with vertical force during the first part of the movement. After the peak, grip force tended to decrease less than in the slower movements. The pattern of grip force modulation during the initial part of the downward movement is clearly different from the pattern observed at the slower rate. For example, B's grip force did not decrease at the start and A's grip force actually increased. This reflects the fact that the vertical force was reduced below zero. If the grip force was too small at this point, the hand would actually slip downwards past the object. Note that A's minimum vertical force was less than B's. This might explain why A's grip force actually increased during the initial part of the movement, whereas B's remained more or less constant.

The results described thus far demonstrate that in vertical point-to-point arm movements with grasped objects, grip force is modulated in phase with the vertical force acting on the object. The close temporal coupling between grip force and vertical force suggests that grip force is specified in anticipation of changes in vertical force (or load force) that are related to the kinematics of arm movement. Anticipatory control of grip force may be contrasted with reflex-mediated changes in grip force. The bottom panel of Fig. 5 illustrates the changes in grip force and vertical force brought about by a brief and unexpected perturbation of the object. The object was lightly tapped with the end of a pencil while the subject (A) held the object with eyes closed. The perturbation caused a small decrease in vertical force. An increase in grip force was seen some $90 \mathrm{~ms}$ later. This is about the time expected for a supraspinal sensorimotor reflex loop to complete (Johansson and Westling 1987; Cole and Abbs 1988). For comparison, the forces during an upward movement are shown in the top panel. In this case, the grip force started to increase at the same time as the vertical force. Note that the change in vertical force at the onset of the upward movement was about twice the 


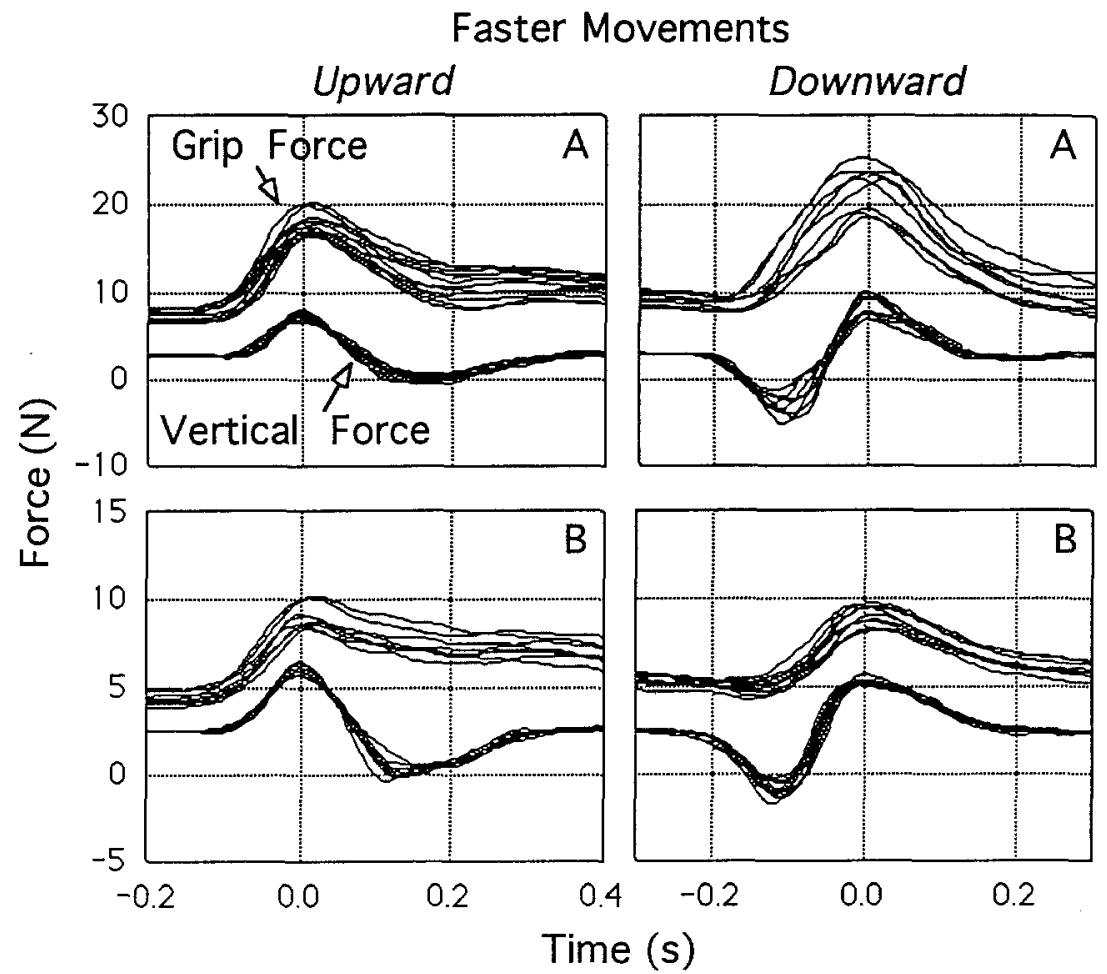

Fig. 4. Grip forces and vertical forces of faster vertical movements produced by subjects $A$ and $B$. In a number of downward trials, the vertical force decreases below zero during the initial part of the movement. This corresponds to an increase in load force. Note that the grip force also increases in these cases
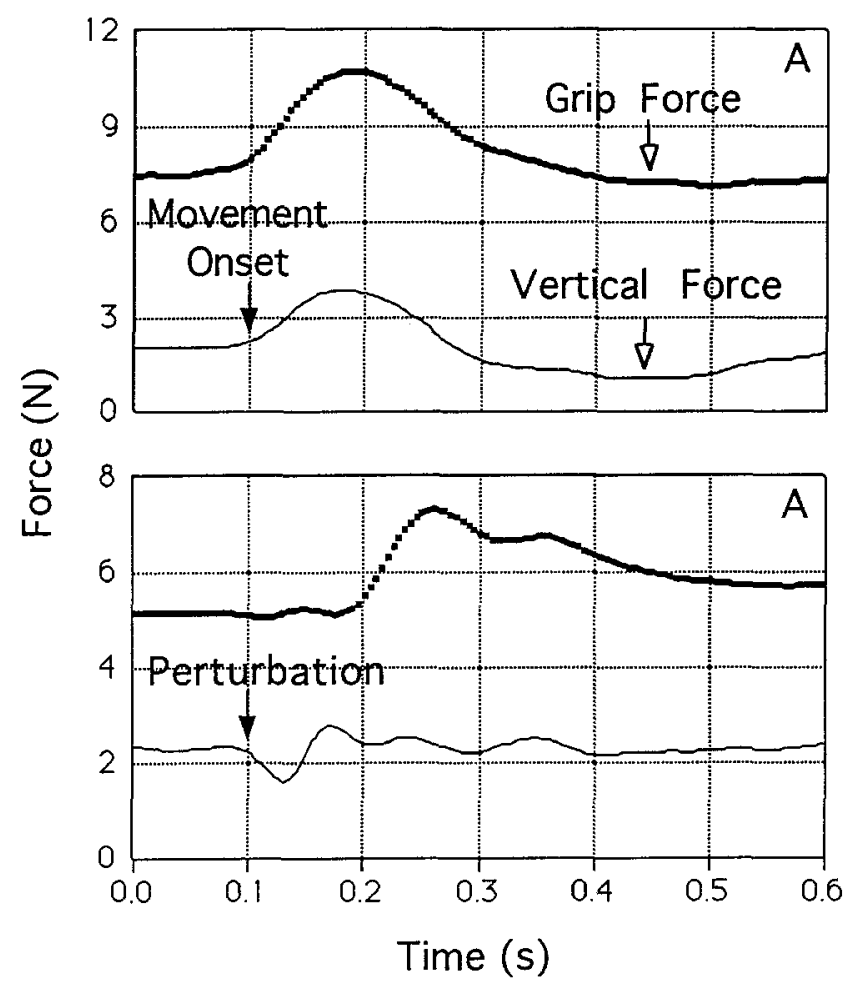

Fig. 5. Grip force response to a brief unexpected change in load force (bottom panel). The change in load force was produced by tapping down on the object with a pencil while the subject $(A)$ grasped it with eyes closed. Grip force starts to increase some $90 \mathrm{~ms}$ after the perturbation was delivered. An upward movement is shown for comparison (top panel). In both cases, at the very onset, the direction of the change in force exerted by the object on the hand was downwards change in vertical force induced by the perturbation. As shown in the bottom panel, a small fluctuation in grip force was seen during the perturbation. However, the change in grip force was much smaller than the subsequent reflexive change in grip force or the change in grip force observed during the upward movement. This indicates that the change in grip force seen during voluntary arm movement is not due to mechanical coupling but reflects neural control.

Quantitative assessment. In order to quantify the coupling between grip force and vertical force, times to maximum grip force were linearly regressed against times to maximum vertical force. In Fig. 6, the time of maximum grip force is plotted against the time of maximum vertical force for two subjects (A and B). The plots include data from slow and fast up and down movements. Strong positive correlations between the times of the grip force and vertical force maxima were observed for both subjects $(r>0.98)$. The slopes of the best fit regression lines were just less than unity ( 0.93 and 0.91 for subjects $A$ and $B$, respectively) and the intercepts were close to zero $(0.02$ for both subjects). This analysis reveals that the grip force and vertical force maxima reliably and closely coincide in time.

While the temporal coupling between grip force and vertical force maxima is very precise, the coupling between the maxima is less so. Figure 7 shows plots of maximum grip force versus maximum vertical force for the same subjects (A and B) shown in Fig. 6. Again, data from vertical movements of varying rate and direction are included in both plots. The correlations between the grip and vertical force maxima ( 0.87 and 0.81 for subjects $\mathrm{A}$ and $\mathrm{B}$ ) are lower than those observed for the times of 

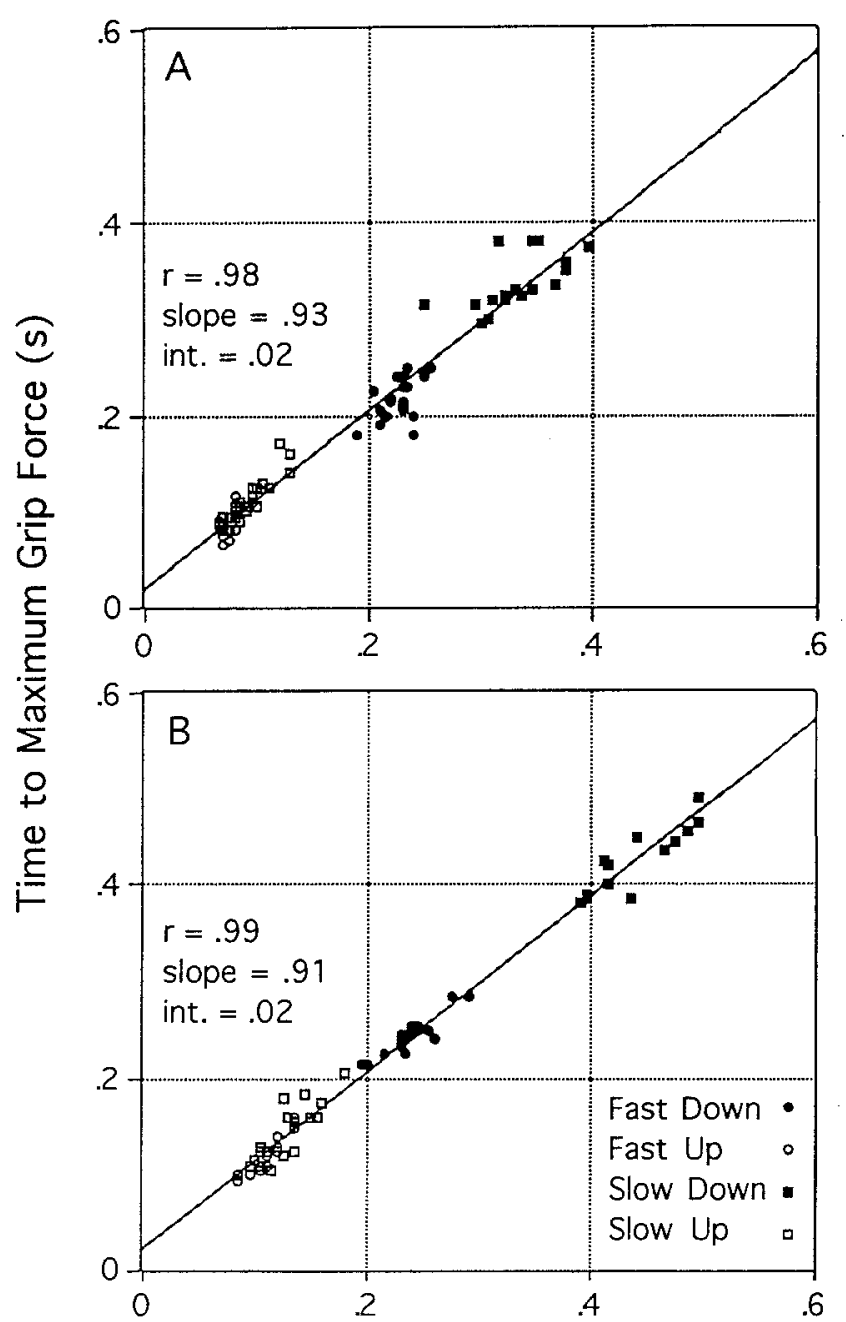

Time to Maximum Vertical Force (s)

Fig. 6. Plots showing the time to maximum grip force versus the time to maximum vertical (or load) force for two subjects ( $A$ and $B$ ). The slopes of the least squares regression lines are close to unity and the intercepts (int.) are close to zero. In addition, the correlation coefficients $(r)$ are very high. This indicates that in vertical movements of varying rate and direction, grip force and vertical force maxima coincide closely in time

these maxima. Inspection of the plots shows that, for a given rate and direction of movement, the correlation between the maxima appears to be weaker. This question will be addressed below.

The strong temporal linkage between grip force and vertical force maxima was seen on the very first trial. In Fig. 8, the time to maximum grip force is plotted against the time to maximum vertical force. The data points are from the initial upward and downward movements of seven subjects performing this task for the first time. The slope of the linear regression line relating the times to grip force and vertical force maxima was slightly less than unity (0.85). This is due to the fact that, in the upward movements, the maximum grip force tended to occur just after the maximum vertical force. A high correlation $(r=0.97)$ between the maxima times was observed.

Figure 9 presents records from experiment 3 , in which subjects were required to make upward movements with

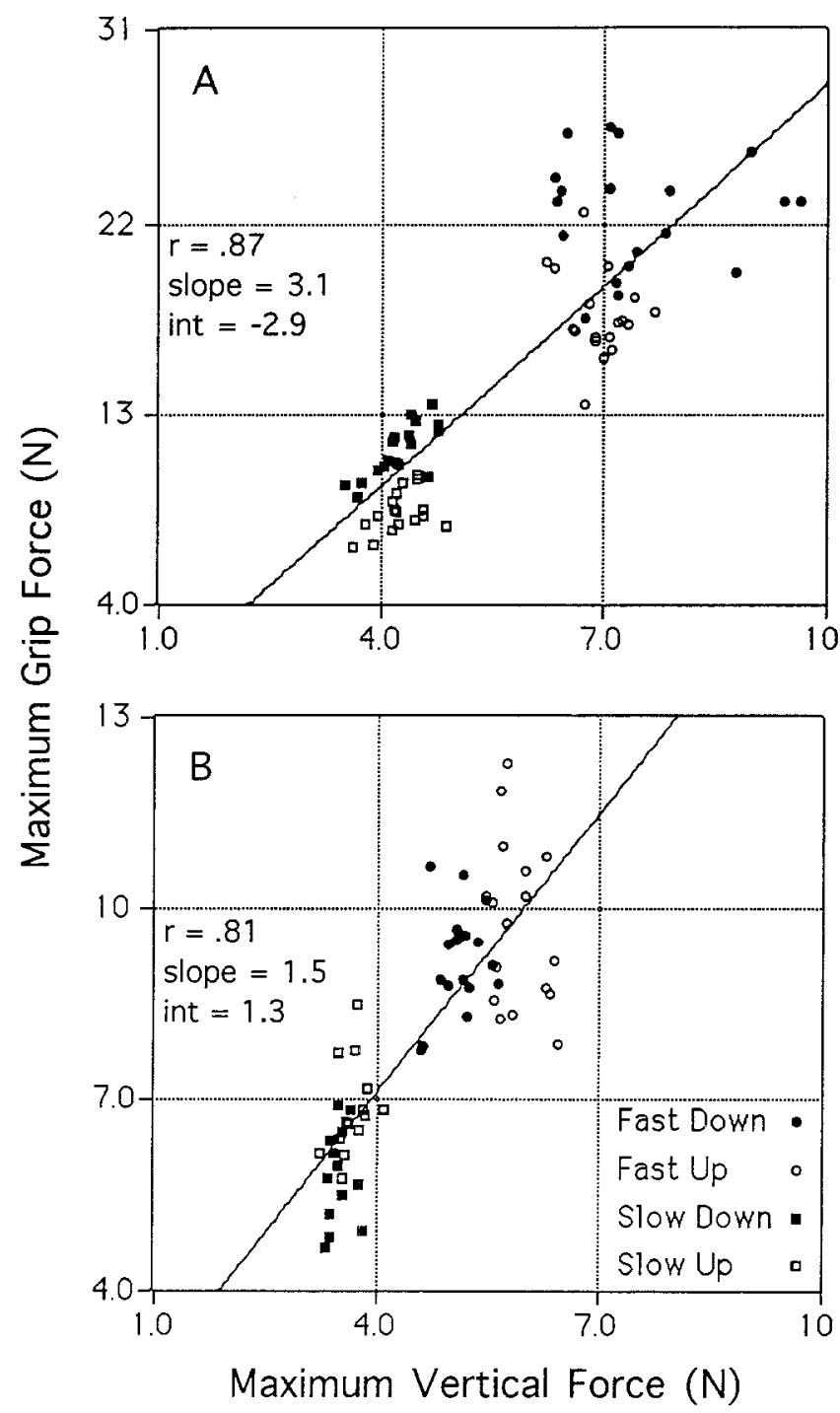

Fig. 7. Plots of maximum grip force versus maximum vertical force. The data are from the same movements from which the data in Fig. 6 were obtained. For both $A$ and $B$, the correlations between the grip force and vertical force maxima are lower than the correlations between the corresponding times

a target maximum vertical force level of 7-9 N. Data from four subjects (A-D) are shown. After each trial, the vertical force was displayed along with the target on a monitor. The target was not varied within subjects. The top panels of the figure show the mean grip force and vertical force functions for four subjects (thick dotted traces) averaged over 20 trials. The thin traces represent the mean plus $1 \mathrm{SD}$. As can be seen, the variability of both the vertical force and grip force functions under these conditions was low. In agreement with the results described above, grip force and vertical force increased together during the first part of the movement and then decreased together. In all four subjects, grip force started to rise just before the vertical force. Johansson and Westling (1984) have shown that grip force also starts to increase ahead of load force in object lifting. In their task, the grasp had to be formed before lifting and, thus, an early increase in grip force was expected. In contrast, in 
the present task, the grasp is already formed at the start of the movement. Nevertheless, grip force began to increase before the vertical force. Note also that the maximum grip force occurs, on average, some $5-10 \mathrm{~ms}$ after the maximum vertical force in all subjects. It may be noted that, in these movements, the correlations between maximum grip force and maximum vertical force were substantially lower (the correlation coefficients ranged from $r=0.1$ to $r=0.63$ ) than those reported above for vertical movements of varying rate and direction. This

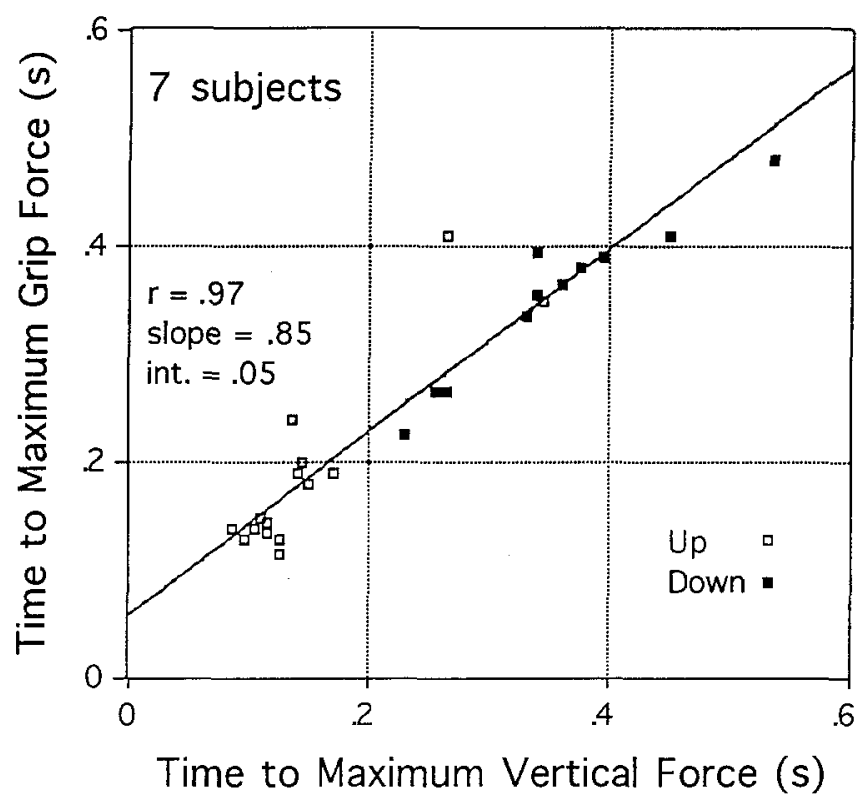

Fig. 8. Plot of the time to the maximum grip force versus the time to the maximum vertical force for vertical movements. The data are from the very first trials of seven subjects. Note the high correlation between times of the grip force and vertical force maxima presumably reflects the reduced range of maximum load force values used in the correlation. Although little variation in time to maximum load force values was observed in this task, the correlations between the times of the grip and load force maxima were, as before, very high $(r>0.98$ for all four subjects).

\section{Horizontal movements}

A number of similarities were observed between vertical and horizontal movements. In both, grip force increased during the movement in anticipation of load force changes. Although the pattern of grip force modulation differed in the vertical and horizontal, this may have been related to differences in load force variation. For example, whereas horizontal movements typically exhibit similar peaks in load force during the initial and final phases, vertical movements feature a predominant load force peak because of the offset in load force due to gravity. Indeed, we will show that, in both vertical and horizontal movements, changes in grip force are quite closely coupled to modulations in load force.

Figure 10 shows grip force, load force and inertial force records for a number of medial and lateral movements performed at the slower rate by subjects $A$ and $B$. Recall that load force represents the magnitude of the resultant force. In order to maintain a stable grip, the frictional force developed by gripping must counteract the load force. Several points may be emphasized. Regardless of the direction of movement, grip force increased at the start together with load force. The grip force remained elevated during the movement and then decreased towards the end. In a number of cases, two distinct grip force peaks were discerned which coincided with the two peaks in load force. In other trials, a "bulge"

\section{Upward Movements to Peak Load Force Targets}

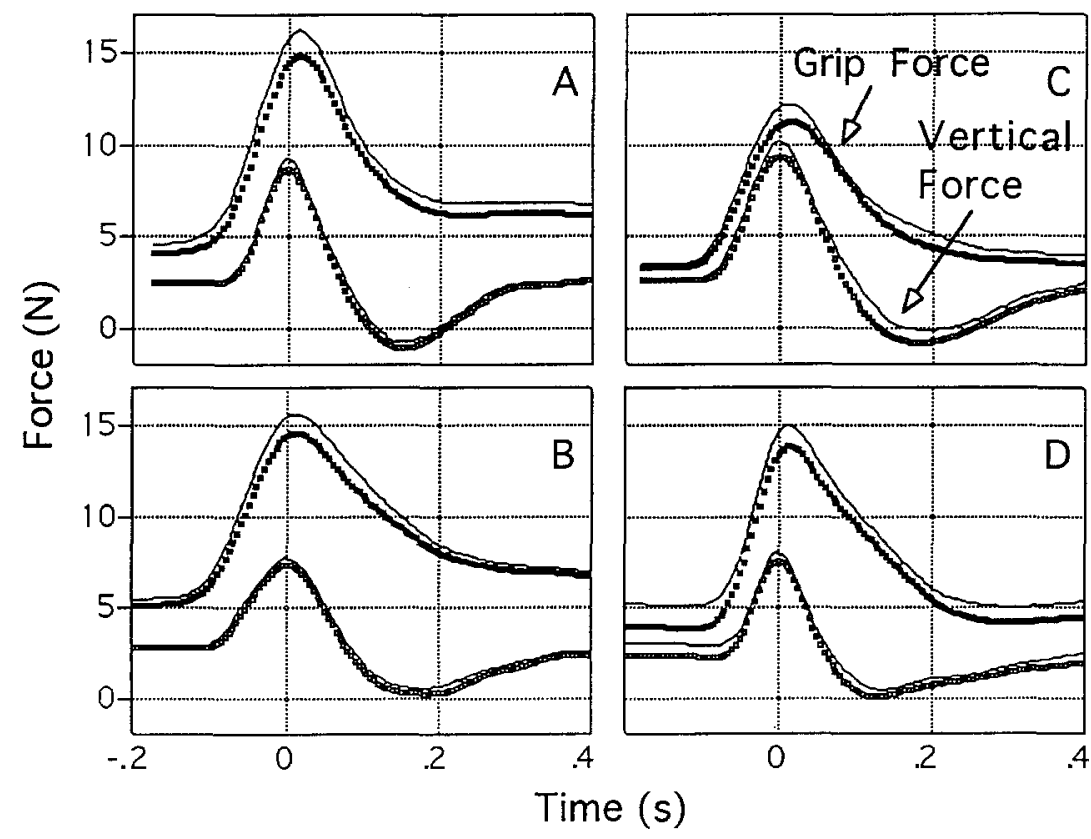

Fig. 9. Average grip force and vertical force records $(n=20)$ for fast upward movements "aimed" at a maximum vertical force target. Data from four subjects are shown $(A-D)$. The thin traces are $1 \mathrm{SD}$ above the mean. The maximum mean grip force occurs some 5-10 ms after the maximum mean vertical force. In addition, the mean grip force starts to increase just ahead of the mean vertical force 


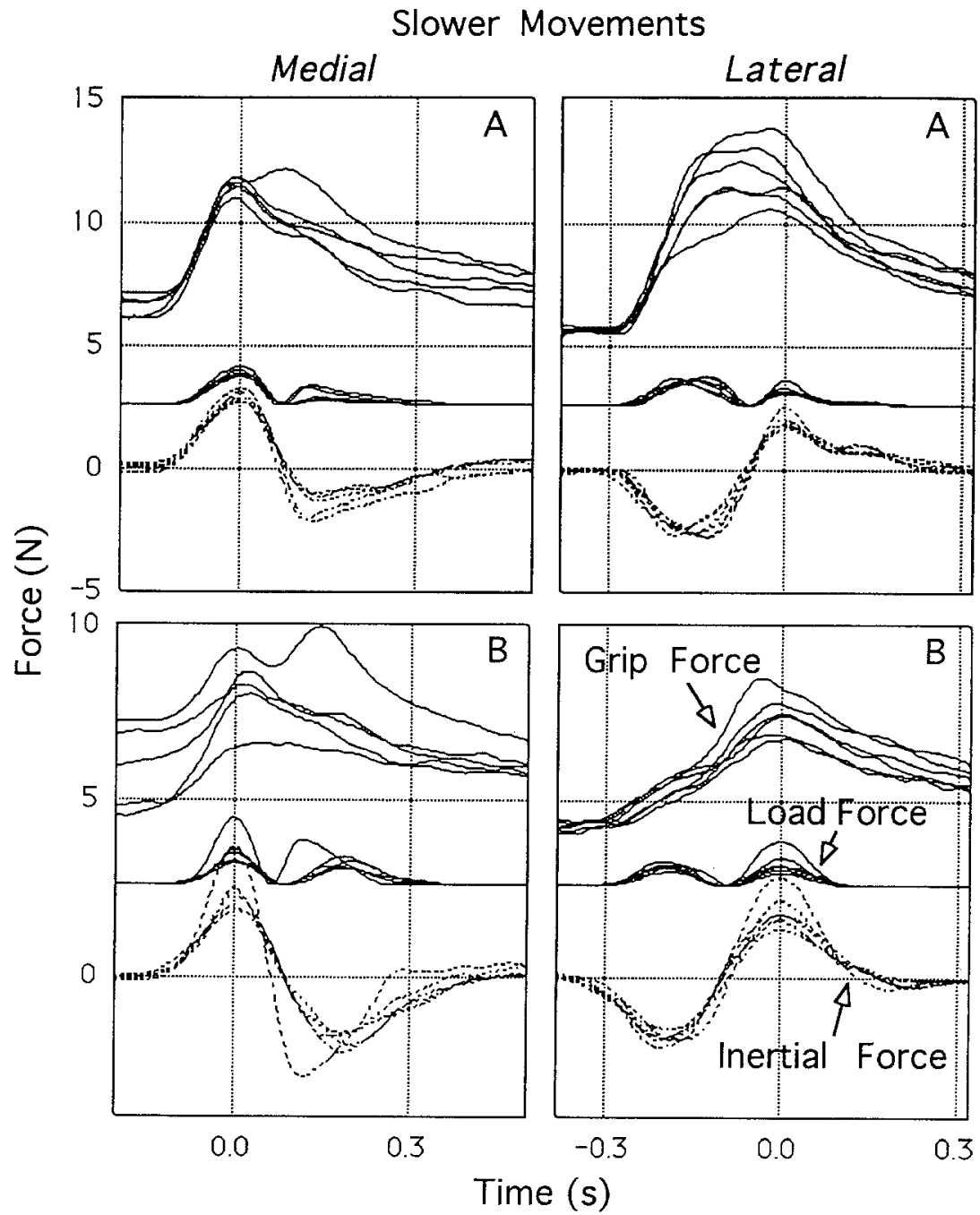

Fig. 10. Grip force, load force and inertial force records for slower horizontal movements of two subjects ( $A$ and $B$ ). Grip force increases at the start of the movement and remains elevated until near the end. Note that peaks in load force occur both near the start and towards the end of the movement. In a number of trials, two grip force peaks can be seen that coincide with the peaks in load force or inflection in grip force was detected which corresponded in time with a load force peak. Even when two peaks in grip force were observed, the grip force only dipped slightly in between them. On the other hand, load force dropped back to its initial level between peaks. Note that it would seem to be unnecessary for the grip force to slavishly vary with load force at this juncture in the movement. The fact that it didn't suggests that the control of grip force during movement is both anticipatory and flexible.

Grip, load, and inertial forces recorded during faster medial and lateral movements are shown in Fig. 11 for the same two subjects. In both directions, grip force started to increase at the onset of the movement and stayed high until near the end, as was observed at the slower rate. In many trials, the grip force function featured two distinct peaks that coincided with the load force peaks. In other trials, with a single grip force peak aligned with one of the load force peaks, there was an inflection point in grip force that coincided with the other load force peak. Note that only A's medial movements never exhibited twin grip force peaks. This may reflect the fact that the second load force peak in these trials was always smaller than the first. Note also that there was not a consistent effect (across the two subjects) of direction on the time of maximum grip force as there was for the slower movements.

As reported above, in vertical movements, the times of grip force and vertical force maxima were highly correlated. In order to assess timing in horizontal movements, we correlated the time of maximum grip force with the time of the nearest peak in load force (data from movements of varying rate and direction were combined, as was done for the vertical movements). The correlation coefficients were very high for both $\mathrm{A}(r=0.96)$ and $\mathrm{B}(r=0.99)$ and are comparable with the values found for the same subjects in vertical movements ( $r \geq 0.98$ for each subject). The slopes of the least squares regression lines relating time of maximum grip force to time of the nearest load force peak were close to unity for A $(0.90)$ and B $(0.99)$. This indicates that the two peaks occurred at about the same time. We also correlated the maximum grip force with the nearest peak load force. Lower correlation coefficients were obtained for both A $(r=0.87)$ and B $(r=0.93)$. Again, comparable values were found for the vertical movements. Thus, in both vertical and horizontal movements, extremely tight correlations between the times of grip force and load force peaks were observed. However, the linkage between corresponding peak force values was somewhat weaker. 


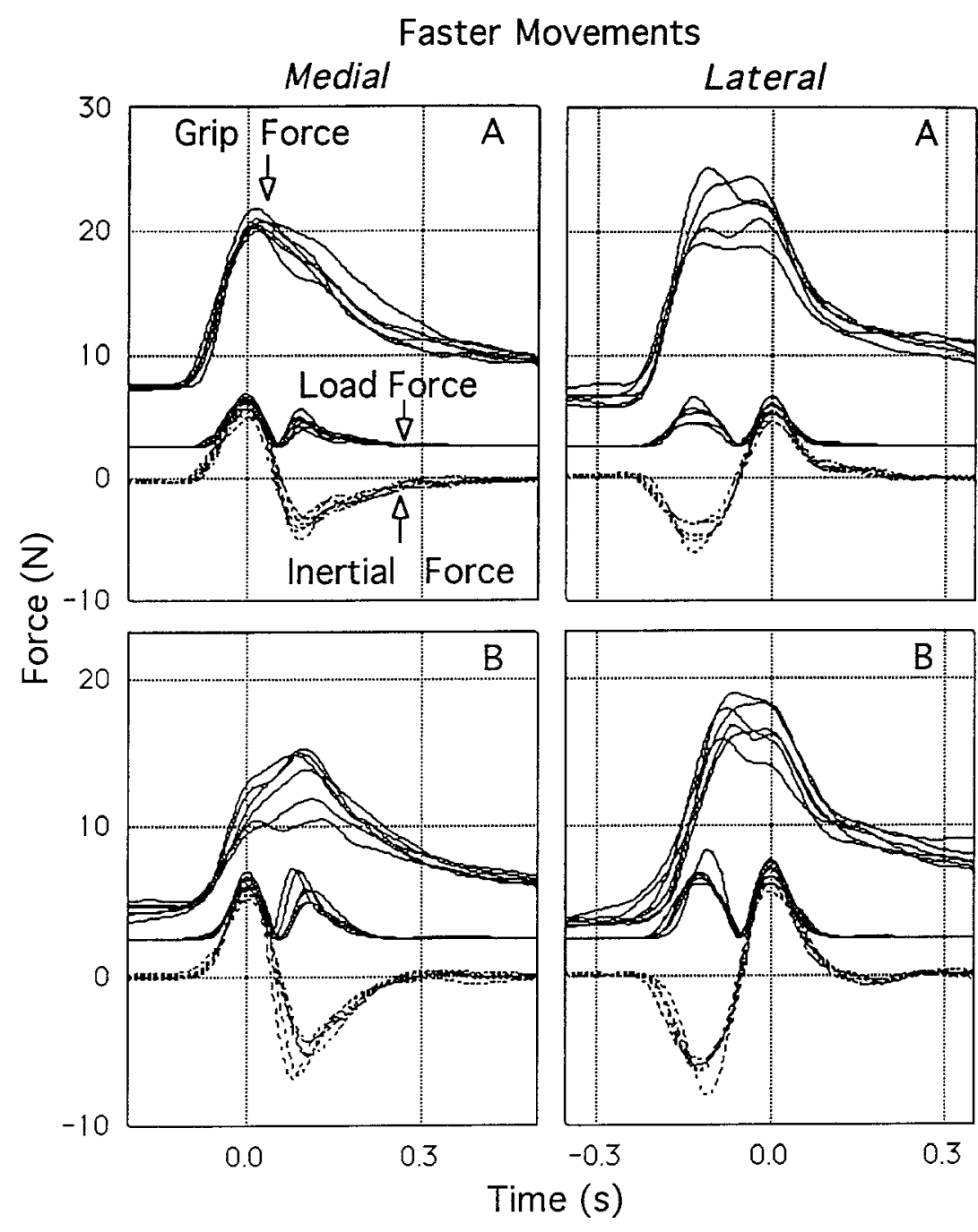

Fig. 11. Grip, load and inertial force records for faster horizontal movements produced by subjects $A$ and $B$. The pattern of grip force modulation is similar to that observed at slower rates. The grip force rises at the start of the movement and stays high until near the end. Two grip force peaks, coinciding with the two peaks in load force, can be seen in a number of trials
Table 1. Means and SDs of three ratios computed for vertical and horizontal movements

\begin{tabular}{|c|c|c|c|c|c|c|}
\hline & \multicolumn{3}{|c|}{ Subject A } & \multicolumn{3}{|c|}{ Subject B } \\
\hline & Mean & $\mathrm{SD}$ & $n$ & Mean & $\mathrm{SD}$ & $n$ \\
\hline \multicolumn{7}{|c|}{ Elevation ratio $(\mathrm{ER})$} \\
\hline Vertical & 0.42 & 0.13 & $24 * *$ & 0.36 & 0.12 & $40^{* *}$ \\
\hline Horizontal & 0.55 & 0.08 & 40 & 0.55 & 0.14 & 40 \\
\hline \multicolumn{7}{|c|}{ Ratio of max. grip force to nearest peak load force (GL) } \\
\hline Vertical & 2.63 & 0.56 & $24 * *$ & 1.77 & 0.21 & $40^{* *}$ \\
\hline Horizontal & 2.97 & 0.70 & 40 & 2.34 & 0.35 & 40 \\
\hline \multicolumn{7}{|c|}{ Ratio of max. grip force to nearest peak inertial force (GI) } \\
\hline Vertical & 5.22 & 1.16 & $24 *$ & 4.45 & 1.45 & $40 * *$ \\
\hline Horizontal & 4.64 & 1.11 & 24 & 3.23 & 0.72 & 40 \\
\hline
\end{tabular}

* $P<0.1$; ** $P<0.001$; ANOVA

\section{Comparisons between vertical and horizontal movements}

To test, quantitatively, the idea that grip force tends to be elevated for a longer period of time in horizontal movements than in vertical movements, we computed an elevation ratio (ER). We took the time at which the grip force decreased half way from the peak to the final grip force minus the time at which the grip force increased half way between the initial grip force and the peak and divided this by the duration of the movement. ER means and SDs are presented in Table 1 for two subjects treated separately. ANOVA was carried out to test for differ- 
ences between vertical and horizontal movements. As expected, ER was significantly greater in horizontal movements for both A $\left(F_{1,63}=20.0 ; \quad P<0.001\right)$ and B $\left(F_{1,79}=44.4 ; P<0.001\right)$. (Note that data from movements of varying rate and direction were combined.) These results support the observation that grip force tends to be elevated during both the acceleratory and deceleratory phases of horizontal movements where peaks in load force are seen in the two phases.

In horizontal movements, the amplitude of inertial force modulation was greater than the amplitude of load force modulation. In contrast, in vertical movements, inertial and load forces modulated to the same extent. Thus, a horizontal movement with the same degree of inertial force modulation as a given vertical movement would have had a smaller degree of load force modulation. The question arises as to whether the changes in grip force in vertical and horizontal movements are more sensitive to modulations in inertial force or load force. To examine this issue, we computed two ratios: the ratio of maximum grip force to the nearest peak load force (GL) and the ratio of maximum grip force to the absolute value of the nearest peak inertial force (GI). We used the absolute value of the inertial force because we were interested in the amplitude of modulation rather than the direction. Note that in both vertical and horizontal movements, the "nearest" peak in load force (or inertial force) always coincided closely in time with the maximum grip force.

If grip force modulation is more sensitive to changes in load force, then we would expect GL to be similar for vertical and horizontal movements. On the other hand, if modulation in grip force is more sensitive to inertial force changes then we would expect GI to be similar for movements in the vertical and horizontal. The results are presented in Table 1. GL was significantly higher for horizontal movements for both $\mathrm{A}\left(F_{1,63}=39.6 ; P<0.001\right)$ and $\mathrm{B}\left(F_{1,79}=79.9 ; P<0.001\right)$. This finding suggested to us that grip force modulation might be more closely linked to changes in inertial force. It may be noted that in the movements that we recorded the amplitude of inertial force modulation was similar in the vertical and horizontal. However, GI was greater in vertical movements for both A $\left(F_{1,63}=3.8 ; P=0.056\right)$ and $\mathrm{B}\left(F_{1,79}=22.5\right.$; $P<0.001$ ), although the difference was significant at the 0.05 level of probability in B only. Nevertheless, this finding indicates that the modulation of grip force relative to changes in inertial force is greater in the vertical. Taken together, these results suggest that the amplitude of changes in grip force during movement is sensitive to some combination of load force and inertial force modulation.

\section{Discussion}

In the Introduction we suggested that one way the system might deal with loads induced by arm movement would be to increase grip force to a steady level throughout the movement. Comparison of grip forces during upward and downward movements clearly indicates that this is not what happens. In upward and downward move- ments, clear grip force maxima are seen during the acceleratory and deceleratory phases, respectively, where load force is maximal. In other words, the timing of maximum grip force depends on the timing of the maximum load force, which in turn depends on the direction of movement. In addition, a reduction of grip force was sometimes observed at the onset of downward movements where load force decreases towards zero. Although the steady grip force after the movement was sometimes higher than before (for example, see Fig. 4, data for subject $B$ ), the change in force was small in comparison with the large phasic fluctuations in grip force related to inertial loading. These findings indicate that grip force is modulated over time with load force and is not merely raised during movement irrespective of the pattern of load force variation.

In horizontal movements, grip force started to increase at the onset and remained elevated. However, because peaks in load force occurred during both the initial acceleratory and subsequent deceleratory phases, elevated grip forces throughout the movement were not unexpected. In many trials, separate grip force peaks were observed and these coincided with the peaks in load force. This indicates that even when grip force is elevated during most of the movement, it is not the case that grip force is simply set at a constant level. Thus, in horizontal as in vertical movements, we found that grip force was modulated in phase with load force. It will be interesting, in future work, to examine patterns of grip force modulation in longer duration movements with a constant velocity phase (Cooke and Brown 1990). During the constant velocity phase, we might expect grip force to drop back towards the initial pre-movement level, since inertial forces related to acceleration would be greatly reduced. On the other hand, the grip force might remain at least somewhat elevated, reflecting a general "movement" component of grip force.

The temporal coupling between grip force and load force is particularly striking. For example, we have shown that, in both vertical and horizontal movements, maximum grip force occurs at about the same time as the corresponding peak in load force. In contrast, the linkage in terms of force magnitudes is weaker. Correlations between grip force and load force maxima were less than the correlations between the time to maximum grip force and the time to maximum load force. The question arises as to whether similar relations might be manifest in lifting an object.

We have shown that changes in grip force anticipate fluctuations in inertial force (and hence load force) that result from arm movements with a hand-held mass. This finding adds to evidence garnered by Johansson and colleagues (Johansson and Westling 1984; Johansson et al. 1992) that grip force is programmed in advance of voluntary manipulations of mechanically predictable objects. Thus, not only do grip force adjustments anticipate environmental demands imposed by the properties of the object (Johansson and Westling 1984), they also anticipate the consequences of our own actions.

The parallel changes observed in grip and load force suggest that modulations in grip force are planned as 
opposed to being reflexive responses to fluctuations in load force. If changes in grip force during movement were subserved by reflexes, then we would have expected the peak grip force to lag behind the peak load force by some 60-90 ms (Johansson and Westling 1987; Cole and Abbs 1988). We would argue that the coupling between grip force and load force reflects motor commands rather than grasp mechanics. When the object was perturbed unexpectedly, grip force did not increase until $90 \mathrm{~ms}$ later. If the coupling observed between grip force and load force had been a property of the mechanics of the grasp, then an immediate increase in grip force would have been expected.

What mechanism underlies the tight coupling between grip force and load force? Two ways in which this might occur can be considered. First, commands to hand and arm muscles might be issued in parallel by some high-level control system. In this case, both the arm movement and grip force trajectories would be specified by this system. Second, the commands to the arm movement could be used to drive the commands to the hand. In this scheme, the arm commands would be sent to a "grip force module" that would transform them into grip force commands. This module could, in principle, predict the load force changes resulting from the movement on the basis of the planned arm trajectory. It might also receive inputs relating information about the properties of the object.

Regardless of which scheme might be employed, a striking feature of the coupling between grip and load force is that it is not easily overridden by voluntary control. We observed that, when subjects were asked to keep grip force constant while moving the object, they were unable to do so. This was the case even when the subjects were given visual feedback of grip force and load force during the movement. In an attempt to keep grip force constant, most subjects increased the overall level of grip force. This strategy makes sense, because, in principle, changes in grip force during the movement would not be required to prevent slippage. Nevertheless, we observed that grip force was still modulated in phase with load force, although the amplitude of modulation was reduced.

Coordination between hand grasp and arm movement is also observed in other tasks. For example, anticipatory changes in hand shape while reaching for an object have been found to be temporally linked to the arm movement which transports the hand towards the object (Jeannerod 1981; Wing et al. 1986; Wallace and Weeks 1988, Paulignan et al. 1990; Haggard and Wing 1991). The results reported here document another way in which hand function is temporally coupled to movement of the hand through space. Note that the problem of coordinating grasp and arm movement is also posed in robotics. In pick-and-place tasks, for example, the robot must pick up the object with its end-effector and then move it to another location. However, most of the studies on robotic manipulation have focused on the configuration and stability of different grips under static conditions (Mason and Salisbury 1985) and have not dealt with problems associated with planned motions (Lozano-Perez 1982).
By comparing vertical and horizontal movements, it is possible to assess whether modulations in grip force are more sensitive to changes in the total load force acting on the object or the inertial load alone. We found that the ratio of maximum grip force to the corresponding peak in load force was reliably greater in horizontal movements. However, the ratio of maximum grip force to the corresponding peak in (absolute) inertial force was greater in vertical movements. This suggests that, in point-to-point arm movements, modulations in grip force may be planned in relation to some combination of load force and inertial force change.

The precise temporal coupling between grip and load force was evident on the first movement trial, indicating that subjects do not have to "learn" this task. This may be a reflection of the regularity with which objects are picked up and moved in everyday activities. The strong linkage between grip force and load force presumably reflects a well-developed and well-tuned synergy that is readily available as soon as it is required. (We are presently carrying out work on repetitive cyclic arm movements to examine how grip force modulation might adapt over time to predictable fluctuations in load force.) The question arises as to when, in the course of development, the synergy appears. Forssberg et al. (1991) have suggested that mature anticipatory precision grip force control does not appear until about 2 years of age. However, children may have found the object lifting task used by these workers to be difficult, as it required coordinated and accurate arm movement (to grasp the object) as well as coordinated grip force control (when lifting). Simply moving a grasped object about may prove to be a somewhat easier task with which to study the coupling of grip force and load force in development.

\section{References}

Bouisset S, Zattara M (1987) Biomechanical study of the programming of anticipatory postural adjustments associated with voluntary movement. J Biomech 20:735-742

Cole KJ, Abbs JH (1988) Grip force adjustments evoked by load force perturbations of a grasped object. $J$ Neurophysiol 60:1513-1522

Cooke JD, Brown SH (1990) Movement-related phasic muscle activation. II. Generation and functional role of the triphasic pattern. J Neurophysiol 63:465-472

Flanagan JR, Tresilian J, Wing AM (1993) Coupling of grip force and load force during arm movements with grasped objects. Neurosci Lett 152:53-56

Forssberg H, Eliasson AC, Kinoshita H, Johansson RS, Westling G (1991) Development of human precision grip. I. Basic coordination of force. Exp Brain Res 85:451-457

Haggard P, Wing AM (1991) Remote responses to perturbation in human prehension. Neurosci Lett 122:103-108

Horak FB, Esselman P, Anderson ME, Lynch MK (1984) The effects of movement velocity, mass displaced, and task certainty on associated postural adjustments made by normal and hemiplegic individuals. J Neurol Neurosurg Psychiatry 47:10201028

Jeannerod M (1981) Intersegmental coordination during reaching for natural objects. In: Long J, Baddeley AD (eds) Attention and performance, vol IX. Erlbaum, Hillsdale, N.J., pp 153-169

Johansson RS, Westling G (1984) Roles of glabrous skin receptors and sensorimotor memory in automatic control of precision 
grip when lifting rougher or more slippery objects. Exp Brain Res 56:550-564

Johansson RS, Westling G (1987) Signals in tactile afferents from the fingers eliciting adaptive motor responses during precision grip. Exp Brain Res 66:141-154

Johansson RS, Westling G (1988) Coordinated isometric muscle commands adequately and erroneously programmed for the weight during lifting tasks with precision grip. Exp Brain Res $71: 59-71$

Johansson RS, Riso R, Hager C, Backstrom L (1992) Somatosensory control of precision grip during unpredictable pulling loads. Exp Brain Res 89:181-191

Lozano-Perez T (1982) Task planning. In: Brady M, Hollerbach JM, Johnson TL, Lozano-Perez T, Mason MT (eds) Robot motion: planning and control. MIT, Cambridge, Mass., pp 473-498
Mason MT, Salisbury, JK (1985) Robot hands and the mechanics of manipulation. MIT, Cambridge, Mass.

Paulignan Y, Mackenzie C, Marteniuk R, Jeannerod M (1990) Selective perturbation of visual input during prehension movements. Exp Brain Res 83:502-512

Smeets JBJ, Erkelens CJ, Denier van der Gon JJ (1990) Adjustments of fast goal-directed arm movements in response to an unexpected inertial load. Exp Brain Res 81:303-312

Wallace SA, Weeks, DL (1988) Temporal constraints in the control of prehensile movement. J Mot Behav 20:81-105

Wing AM, Turton A, Fraser C (1986) Grasp size and accuracy of approach in reaching. $\mathrm{J}$ Mot Behav 18:245-260 